\title{
Fluorescence of the tri-cyclic adenine and isoguanine derivatives and their ribosides: possible analytical applications*
}

\author{
Alicja Stachelska-Wierzchowska ${ }^{\square}$ and Jacek Wierzchowski \\ Department of Physics and Biophysics, University of Warmia and Mazury in Olsztyn, Olsztyn, Poland
}

\begin{abstract}
Fluorescent tri-cyclic purine analogs, derivatives of isoguanine and adenine, were examined as potential substrates of purine-nucleoside phosphorylase. It was found previously that etheno- derivatives of both compounds are ribosylated in phosphate-free media, but ribosylation places in some instances differ from purine N9. New ribosides are examined as potential substrates of human blood PNP and indicators of this enzyme. Of these, $\mathrm{N}^{6}$-riboside of $1, \mathrm{~N}^{6}$-etheno-adenine was found the most promising.
\end{abstract}

Key words: nucleobase/nucleoside analogs; fluorescence; purine nucleoside phosphorylase; enzyme activity, blood lysates

Received: 18 October, 2019; revised: 13 November, 2019; accepted: 17 November, 2019; available on-line: 05 December, 2019

छe-mail: alicja.stachelska@uwm.edu.pl

*Acknowledgements of Financial Support:

The costs of the article published as a part of the 44th FEBS Congress Kraków 2019 - From molecules to living systems block are financed by the Ministry of Science and Higher Education of the Republic of Poland (Contract 805/P-DUN/2019).

This work was supported by NCN (National Centre of Science) of Poland, the MINIATURA grant \#DEC-2017/01/X/ST5/00807

Abbreviations: EAde, ethenoadenine, 1,N6-ethenoadenine (imidazo[2,1-i]purine); $\varepsilon A d o$, etheno-adenosine, 1,N6-ethenoadenosine; isoGua, isoguanine, 2-oxoadenine; isoGuo, isoguanosine, 2-oxoadenosine; हisoGua, 1,N6-etheno-isoguanine (IUPAC name: 5-oxo-imidazo[1,2-i]purine); PNP, purine-nucleoside phosphorylase; EDTA, ethylenediamine-tetraacetic acid

\section{INTRODUCTION}

Tri-cyclic analogs of the canonical purines are frequently used as fluorescent probes in enzymological research. The best known example is 1,N6-etheno-adenosine ( $\varepsilon$ Ado) and analogs (Leonard, 1984; Leonard, 1985), but other fluorescent derivatives are also known (Virta et

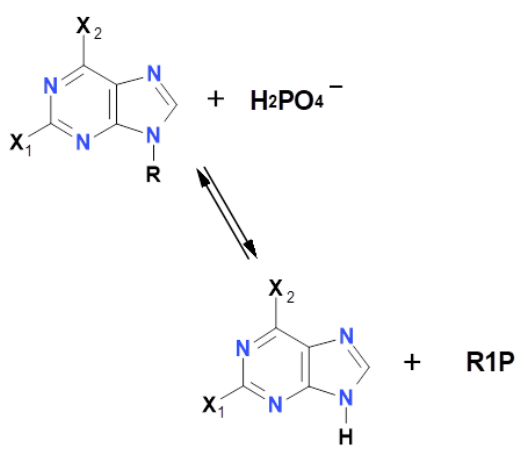

Scheme 1. General course of the reaction catalyzed by purinenucleoside phosphorylase (R, $\beta$-D-ribose; R1P, a-D-ribose-1phosphate). al., 2004; Wang et al., 2017). Tri-cyclic analogs and their ribosides are characterized by moderate biological activity, but some of them reveal promising anti-viral properties (Janz-Wechmann et al., 2015). They are known to react with many enzymes of purine metabolism (Leonard, 1984), and are important intermediates in the process of chemical mutagenesis induced by vinyl chloride (Singer \& Kuśmierek, 1982; Chatterjee \& Walker, 2017).

In our laboratory we are working on an enzyme purine-nucleoside phosphorylase (PNP, E.C. 2.4.2.1, see Scheme 1), responsible for the regulation of the nucleoside concentrations within the living cells, and a target of many types of pharmaceutical interventions, including gene therapy of the inherited immunological disorders (Bzowska et al., 2000; Grunebaum et al., 2013). We have shown that PNP isolated from E. coli, which is known to possess a broad specificity toward various base and nucleoside analogs (Bzowska et al., 2000; Yehia et al., 2017 ) is also active towards tri-cyclic $\varepsilon$ Ado and its 2-aza analog (Wierzchowski et al., 2017). We have found also that $1, \mathrm{~N}^{6}$-etheno-adenine ( $\left.\varepsilon \mathrm{Ade}\right), 1, \mathrm{~N}^{2}$-etheno-guanine and $1, \mathrm{~N}^{6}$-etheno-isoguanine (cisoGua) are good substrates for PNP from E. coli (Fig. 2, left) in the reverse (synthetic) pathway, with catalytic and Michaelis' constants sometimes comparable to those obtained for the enzymatic ribosylation of the parent guanine (StachelskaWierzchowska et al., 2018; Stachelska-Wierzchowska et al., 2019). The ribosides obtained in these reactions are

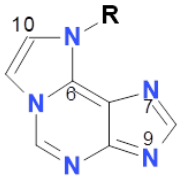

(1)

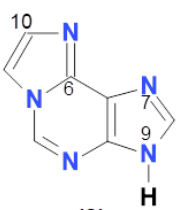

(3)

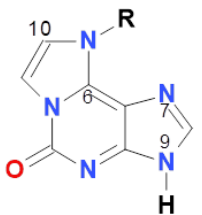

(2)

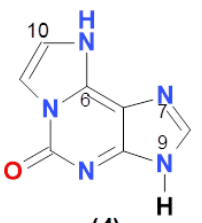

(4)
Scheme 2. Structure of artificial PNP substrates examined in this work ( $R$, ribose).

(1) N6- $\beta$-D-ribosyl-1, $\mathrm{N}^{6}$-ethenoadenine, (2) N6- $\beta$-D-ribosyl-1, $\mathrm{N}^{6}-$ ethenoisoguanine, and products of their phosphorolysis: (3) 1, N6ethenoadenine and (4) 1, $\mathrm{N}^{6}$-ethenoisoguanine. Note that the purine numbering is applied. 
not necessarily identical with those produced chemically, with ribosylation sites varying for different PNP forms.

The purpose of the present paper is examination of substrate properties of new fluorescent ribosides toward the human blood enzyme, and their analytical potential in clinical investigations. We have previously identified two such substrates, isomeric ribosides of 2,6-diamino8-azapurine (Wierzchowski et al., 2014), and now extend our work to include tri-cyclic ribosides with different spectral characteristics.

Human PNP, as well as other mammalian forms of this enzyme, belongs to the second (trimeric) class within the broad family of PNP (Bzowska et al., 2000), and its substrate specificity is different than that of the hexameric (bacterial) forms. In particular, the trimeric forms of PNP are inactive towards adenosine and some derivatives (Bzowska et al., 2000; Yehia et al., 2017). We therefore considered only those ribosides, which were previously shown to be substrates for the calf enzyme (Stachelska-Wierzchowska et al., 2018, 2019). We have chosen some non-canonical ribosides of etheno-adenine and etheno-isoguanosine (Scheme 2).

\section{MATERIALS AND METHODS}

Synthesis of the 1, $\mathrm{N}^{6}$-etheno-isoguanine (base, 4) from isoguanine and chloroacetaldehyde has been described previously (Stachelska-Wierzchowska et al., 2019). This reaction is slow (ca. 7 days at room temperature) but the reaction product crystallized easily from neutralized medium and the reaction yield $(\sim 70 \%)$ was sufficient. Chemo-enzymatic syntheses and identification of two described ribosides of $1, \mathrm{~N}^{6}$ etheno-adenine and 1, $\mathrm{N}^{6}$-etheno-isoguanine (1 and 2 ) were described elsewhere (Stachelska-Wierzchowska et al., 2018; Stachelska-Wierzchowska et al., 2019). These compounds were stored as frozen solutions. Recombinant calf spleen PNP was obtained from Prof. Agnieszka Bzowska (Warsaw University, Poland).

Blood samples were obtained as leftovers from glucometric measurements of one of the authors (J.W.). $10 \mu \mathrm{L}$ blood samples were lysed in $0.5 \mathrm{~mL}$ of $2 \mathrm{mM}$ phosphate buffer, $\mathrm{pH} 7$, containing $0.5 \mathrm{mM}$ EDTA and an aliquot $(\sim 0.2 \mathrm{mM})$ of dithiothreitol. The hemolysates were kept at $5^{\circ} \mathrm{C}$.

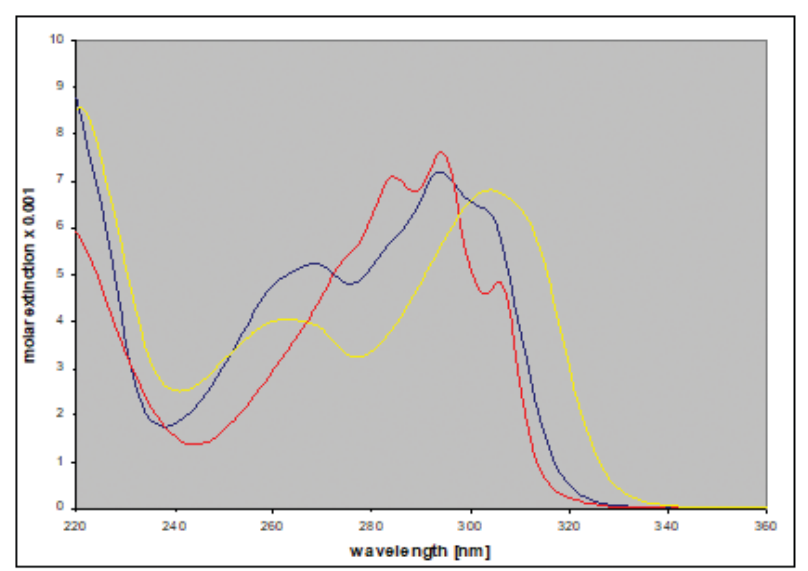

Fluorescence spectra were measured on a Varian Eclipse instrument (Varian Corp., Palo Alto, CA, USA). Spectral resolution was typically $5 \mathrm{~nm}$ (emission path) and $2.5 \mathrm{~nm}$ (excitation path). UV absorption and kinetic experiments were performed on a Cary 5000 (Varian) thermostated spectrophotometer. Enzymatic reactions were carried out at $25^{\circ} \mathrm{C}$. Fluorescence yields were determined relative to tryptophan $(0.15)$ or $1, \mathrm{~N}^{6}$-ethenoadenosine in water (0.56; Leonard, 1984). Spectra were measured in semi-micro $1 \mathrm{~mL}$ cuvettes, pathlength $4 \mathrm{~mm}$, to diminish the inner-filter effect and hemoglobin absorption. Substrate/product concentrations were calculated using known molar extinction coefficients: $8200 \mathrm{M}^{-1} \mathrm{~cm}^{-1}$ for etheno-adenine $(274 \mathrm{~nm})$ and $7000 \mathrm{M}^{-1} \mathrm{~cm}^{-1}$ for etheno-isoguanine at $291 \mathrm{~nm}$ (Stachelska-Wierzchowska et al., 2018; Stachelska-Wierzchowska et al., 2019). Typically, substrate concentrations in fluorescence measurements were 5 -fold lower than those measured by UV absorption.

\section{RESULTS AND DISCUSSION}

It has been shown previously, that enzymatic ribosylation of some nucleobase analogs with PNP as a biocatalyst leads to non-typical ribosides, with ribose moiety attached not necessarily to purine N9, but also to other nitrogen atoms (Stachelska-Wierzchowska et al., 2013; Stachelska-Wierzchowska et al., 2016; StachelskaWierzchowska et al., 2018; Stachelska-Wierzchowska et al., 2019). In particular, while the E. coli PNP directs the ribosyl group predominantly to the N9 of ethenoadenine, the calf enzyme leads to almost exclusively $\mathrm{N}^{6}$ riboside (Stachelska-Wierzchowska et al., 2018). In 1, $\mathrm{N}^{6}$ ethenoisoguanine, the situation is even more complex, since typically mixtures of various ribosides are produced (Stachelska-Wierzchowska et al., 2019).

Spectral properties of new ribosides were presented in previous papers (Stachelska-Wierzchowska et al., 2018; Stachelska-Wierzchowska et al., 2019) and those of three $1, \mathrm{~N}^{6}$-ethenoisoguanine ribosides are summarized in Fig. 1.

The most interesting, from analytical point of view, were those ribosides which were generated by calf spleen $\mathrm{PNP}$, that is, $\mathrm{N}^{6}-\beta$-D-ribosides. We have shown that these ribosides (see Scheme II, above) were also excellent substrates for the E. coli PNP, and phosphorolytic

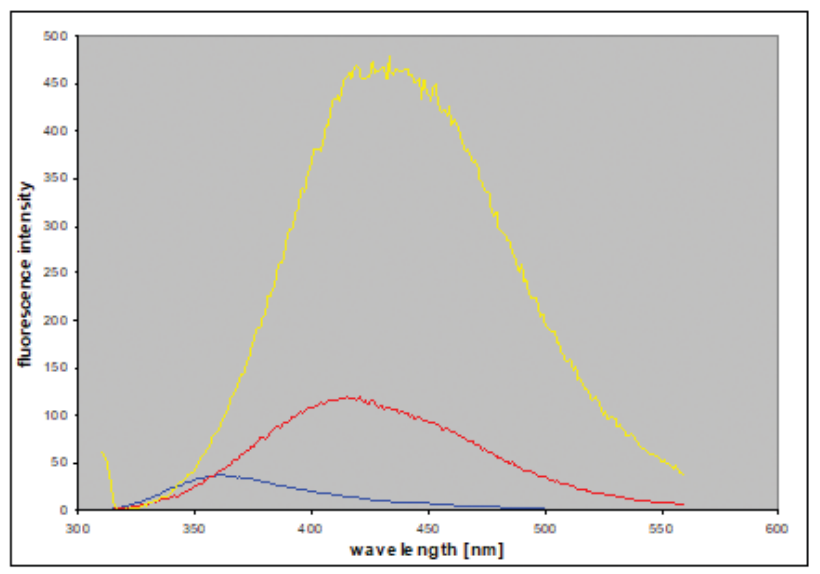

Figure 1. Electronic absorption (left) and fluorescence (right) spectra of three ribosides of 1,N6-etheno-isoguanine, purified by HPLC. Spectra of N9- (red color) and N7- $\beta$-D-ribosides (blue) were measured in $50 \mathrm{mM}$ phosphate buffer, pH 6.5, and that of N6-riboside (2, yellow), at pH 7. The respective quantum yields are 0.34, 0.04 and 0.66. Data from Stachelska-Wierzchowska et al. (Stachelska-Wierzchowska et al., 2019). 

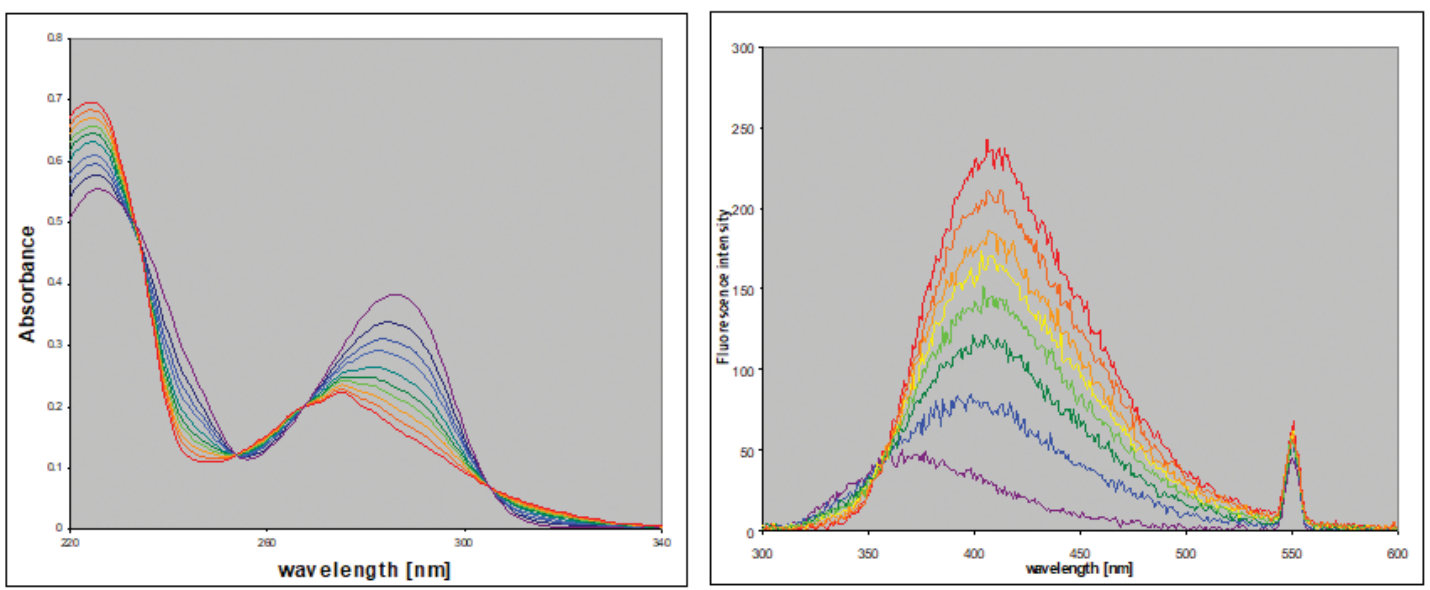

Figure 2. Spectral (left) and fluorescence (right) changes observed during the phosphorolysis of N6- $\beta$-D-ribosyl-1,N6-etheno-adenine (1) with E. coli PNP as a catalyst, in the phosphate buffer, pH 7, at $25^{\circ} \mathrm{C}$.

Initial substrate concentration in UV experiment was $66 \mu \mathrm{M}$, and for emission $13.2 \mu \mathrm{M}$. Cuvettes of a reduced pathlength (4 mm) were used. Fluorescence excitation was at $275 \mathrm{~nm}$. Time intervals: $5 \mathrm{~min}$ for UV absorption, $10 \mathrm{~min}$ for fluorescence. Final curves are drawn in red.
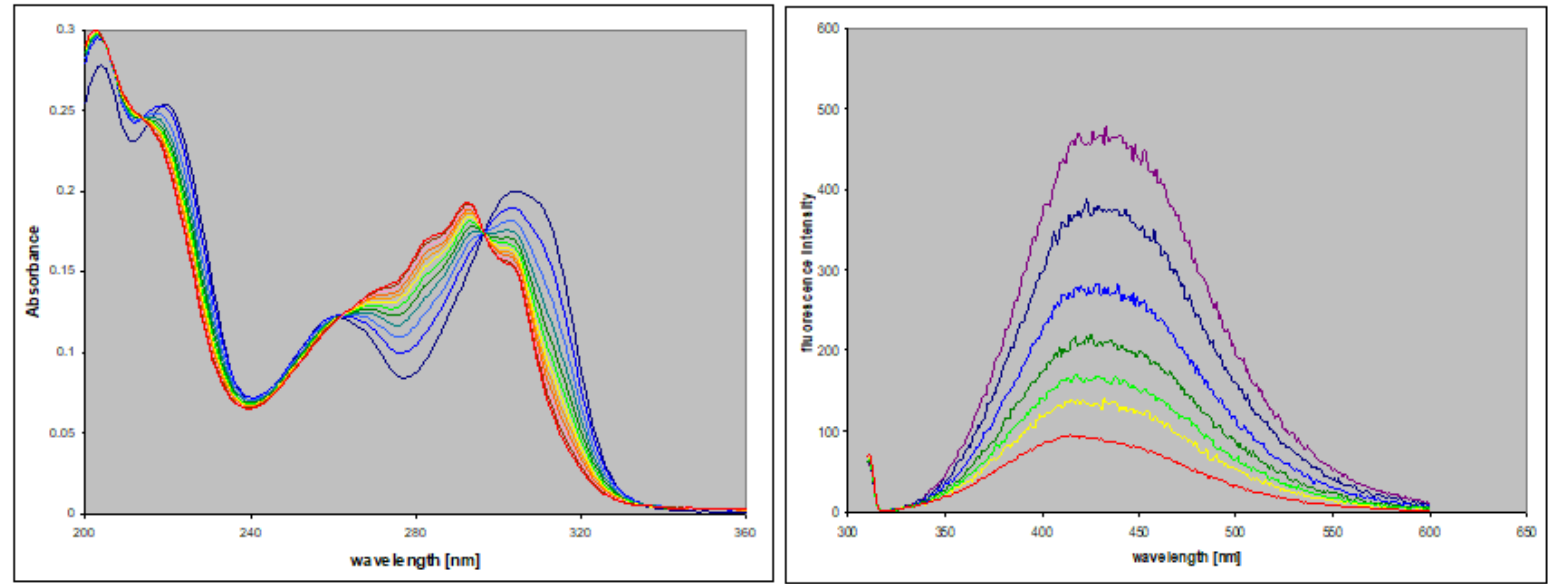

Figure 3. Spectral (left) and fluorescence (right) changes observed during the phosphorolysis of N6- $\beta$-D-ribosyl-1,N6-ethenoisoguanine (2) in the phosphate buffer, $\mathrm{pH} 7$.

Initial substrate concentrations: $68 \mu \mathrm{M}$ for UV, $14 \mu \mathrm{M}$ for emission. Fluorescence was excited at $310 \mathrm{~nm}$. Time intervals are as in Fig. 2. Final curves are drawn in red.
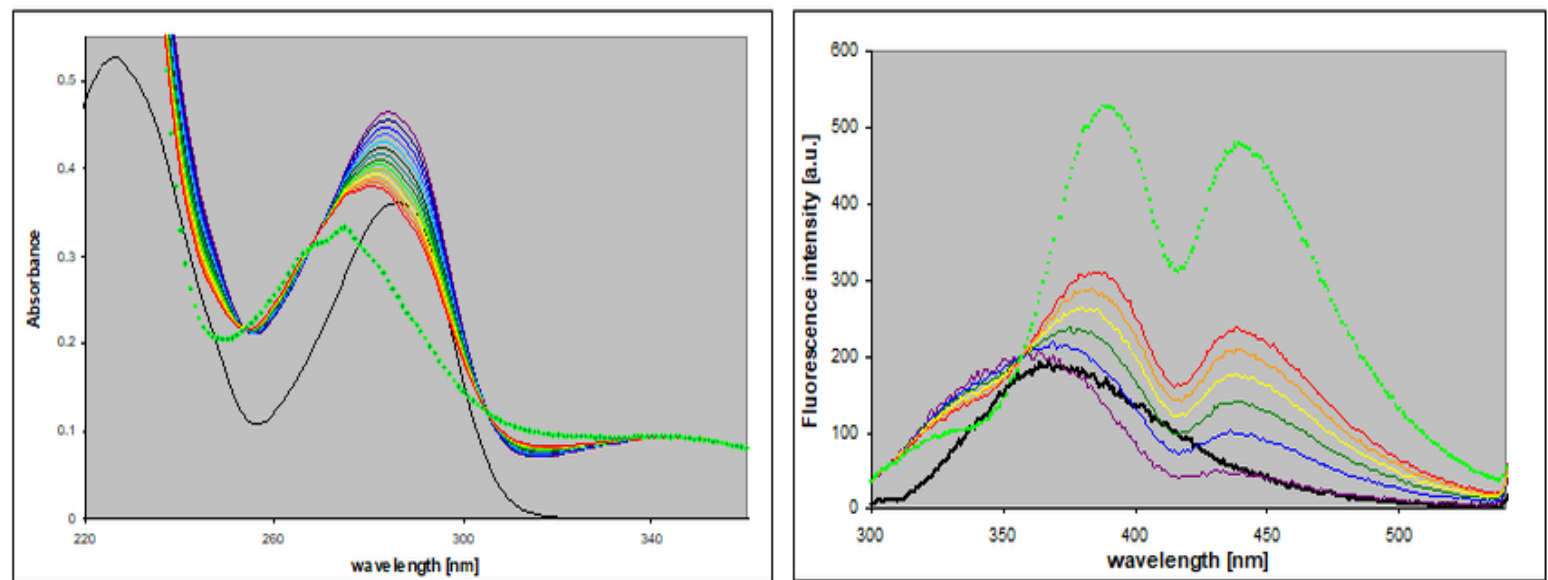

Figure 4. Spectral (left) and fluorescence (right) changes observed during the phosphorolysis of N6- $\beta$-D-ribosyl-1,N6-etheno-adenine (1) with blood lysate as a catalyst, in the phosphate buffer, $\mathrm{pH} 7$, at $25^{\circ} \mathrm{C}$.

Experimental conditions were exactly as in Fig. 2, above. Final curves (points) were obtained by spiking blood sample with the purified E. coli PNP. Black curves refer to purified substrate (1) spectra. Time intervals were 5 minutes for UV absorption, 10 minutes for fluorescence. Fluorescence minimum at $410 \mathrm{~nm}$ is due to light re-absorption by hemoglobin (Slater band). Note that the isosbestic and isoemissive points are strictly maintained. 
reactions were easily followed by UV absorption of fluorescence spectroscopy (Figs. 2 and 3). The new ribosides are fairly stable, and could be stored for months in stock solutions at $-5^{\circ} \mathrm{C}$ (not shown).

Human blood is particularly rich in PNP activity, which is located mainly in erythrocytes (Bzowska et al., 2000). We have shown previously that some phosphorolytic reactions are easily observed spectrally or fluorimetrically using 1000-fold diluted whole blood lysates as a catalyst in ca. $50 \mathrm{mM}$ phosphate buffer (Wierzchowski et al., 2002; Wierzchowski et al., 2014). We have therefore examined the new ribosides as potential substrates for the human PNP, using the same methodology (except optical pathlength of the cuvettes, reduced now to $4 \mathrm{~mm})$.

Experiments with 1000-fold diluted blood lysates has shown that only one of the examined substrates, of $\mathrm{N}^{6}-\beta-\mathrm{D}$-ribosyl-1, $\mathrm{N}^{6}$-etheno-adenine (1), was readily phosphorolysed (Fig. 4) with calculated reaction rate of $\sim 0.45 \mu \mathrm{M} / \mathrm{min}$ at substrate concentration $66 \mu \mathrm{M}$. Phosphorolysis was easily observed spectrophotometrically as well as fluorimetrically. Hemolysate optical background does not interfere with the measurements, except visible re-absorption near the Slater band of hemoglobin at 410 $\mathrm{nm}$ (Fig. 4). Blood proteins contribute somewhat to the overall fluorescence with excitation at $275 \mathrm{~nm}$, but with 1000-fold sample dilution this fluorescence, visible as short-wavelength inflection on Fig. 4, right panel, is low and negligible at $\lambda \sim 430 \mathrm{~nm}$, where the measurements are the most accurate.

The second substrate, the highly fluorescent $\mathrm{N}^{6}-\beta-\mathrm{D}-$ ribosyl-1, $\mathrm{N}^{6}$-etheno-isoguanine (2), was apparently inactive (rate $<0.01 \mu \mathrm{M} / \mathrm{min}$ ) with the same blood sample, although it was rapidly phosphorolysed by the purified E. coli PNP (Fig. 3) as well as by the calf enzyme. This result was somewhat surprising, since the human enzyme belongs to the same class of trimeric PNP, and shows homology of $>70 \%$ with calf PNP (Bzowska et al., 2000). At present, we are unable to explain this phenomenon.

The experimental conditions of the presented reactions need to be optimized, for applications to clinical analyses. This refers particularly to buffer $\mathrm{pH}$, substrate concentration (the apparent $K_{\mathrm{m}}$ for the human enzyme) and excitation wavelength. With conditions fulfilled, the proposed assay will be probably much more sensitive than those described previously.

\section{CONCLUDING REMARKS}

We have described two novel, both fluorescent and fluorogenic, substrates for PNP. One of these, $N^{6}-\beta-D-$ ribosyl-1, $\mathrm{N}^{6}$-etheno-adenine (1), can be used to quantitate PNP activity in human blood. Possible applications include early detection of immunological deficiencies (Grunebaum et al., 2013). The second substrate, N6- $\beta$-Dribosyl-1, $\mathrm{N}^{6}$-etheno-isoguanine (2), can be used to selectively detect bacterial PNP activity in biological samples, with possible use in the investigations of the suicidal gene therapy of cancer, utilizing bacterial PNP to generate in situ cytotoxic nucleobase analogs (Karjoo et al., 2017).

Various assays, including fluorimetric, were previously proposed for this enzyme (Bzowska et al., 2000; Wierzchowski et al., 2002; Wierzchowski et al., 2014), but their sensitivity was not always satisfactory, mostly because of slow reaction rates of the artificial substrates. Therefore search for new, more sensitive substrates is continued.

\section{Acknowledgements}

We thank Prof. Janusz Stępiński for samples of isoguanine and isoguanosine and Prof. Agnieszka Bzowska for enzyme preparations.

\section{REFERENCES}

Bzowska A, Kulikowska E, Shugar D (2000) Purine nucleoside phosphorylases: Properties, functions, and clinical aspects. Pharmacol. Ther. 88: 349-425. https://doi.org/10.1016/S0163-7258(00)00097-8

Chatterjee N, Walker GC (2017) Mechanisms of DNA damage, repair and mutagenesis. Environ. Mol. Mutagen. 58: 235-263. https://doi. org/10.1002/ em.22087

Grunebaum E, Cohen A., Roifman CM (2013) Recent advances in understanding and managing adenosine deaminase and purine nucleoside phosphorylase deficiencies. Curr. Opin. Allergy Clin. Immunol. 13: 630-638. https://doi.org/10.1097/ACI.0000000000000006

Jahnz-Wechmann Z, Framski GR, Januszczyk PA, Boryski J (2015) Bioactive fused heterocycles: Nucleoside analogs with an additional ring. Eur. J. Med. Chem. 97: 388-396. https://doi.org/10.1016/j.ejmech.2014.12.026

Leonard NJ (1984) Etheno-substituted nucleotides and coenzymes: fluorescence and biological activity. CRC Crit. Revs. Biochem. 15: 125199. https://doi.org/10.3109/10409238409102299

Leonard NJ (1985) Adenylates: bound and unbound. Biopolymers 24: 9-28. https://doi.org/10.1002/bip.360240104

Karjoo Z, Chen XG, Hatefi A (2016) Progress and problems with the use of suicide genes for targeted cancer therapy. Adv. Drug Deliv. Rev. 99: 113-128, Part A. https://doi.org/10.1016/j.addr.2015.05.009.

Singer B, Kuśmierek JT (1982) Chemical mutagenesis. Ann. Rev. Biochem. 51: 655-693. https://doi.org/10.1146/annurev.bi.51.070182.003255

Stachelska-Wierzchowska A, Wierzchowski J, Wielgus-Kutrowska B, Mikleušević G (2013) Enzymatic synthesis of highly fluorescent 8 -azapurine ribosides using purine-nucleoside phosphorylase reverse reaction: variable ribosylation sites. Molecules 18: 12587-12598. https://doi.org/10.3390/molecules181012587

Stachelska-Wierzchowska A, Wierzchowski J, Bzowska A, Wielgus-Kutrowska B (2016) Site-selective ribosylation of fluorescent nucleobase analogs using purine-nucleoside phosphorylase as a catalyst: effects of point mutations. Molecules 21: \#44. https://doi. org/10.3390/molecules21010044.

Stachelska-Wierzchowska A, Wierzchowski J, Bzowska A, Wielgus-Kutrowska B (2018) Tricyclic nitrogen base, 1,N6'-Ethenoadenine, and its Ribosides, as Substrates for Purine-Nucleoside Phosphorylases: Spectroscopic and Kinetic Studies. Nucleosides Nucleotides Nucleic Acids 37: 89-101. https://doi.org/10.1080/15257770.2017.1 419255

Stachelska-Wierzchowska A, Wierzchowski J, Górka M, Bzowska A, Wielgus-Kutrowska B (2019) Tri-cyclic nucleobase analogs and their ribosides as substrates of purine-nucleoside phosphorylases. II. Guanine and isoguanine derivatives. Molecules 24: 1493. https:// doi. org/10.3390/molecules 24081493

Virta P, Holmstrom T, Roslund MU, Mattjus P, Kronberg L, Sjoholm R (2004) New nucleoside analogs from 2-amino-9-( $\beta$-D-ribofuranosyl)purine. Org. Biomol. Chem. 2: 821-827. https://doi.org/10.1039/ b316413f

Wang X, Chan KM, Kool ET(2017) Fluorescent nucleobases as tools for studying DNA and RNA. Nature Chem. 9: 1043-1055. https:// doi.org/10.1038/nchem.2859.

Wierzchowski J, Ogiela M, Iwańska B, Shugar D (2002) Selective fluorescent and fluorogenic substrates for purine-nucleoside phosphorylases from various sources, and direct fluorimetric determination of enzyme levels in human and animal blood. Anal. Chim. Acta 472: 63-74. https://doi.org/10.1016/S0003-2670(02)00938-8

Wierzchowski J, Stachelska-Wierzchowska A, Wielgus-Kutrowska B, Mikleušević G (2014) Two fluorogenic substrates for purine-nucleoside phosphorylase, selective for mammalian and bacterial forms of the enzyme. Anal. Biochem. 446: 25-27. https://doi.org/10.1016/j. ab.2013.10.017

Wierzchowski J, Stachelska-Wierzchowska A, Wielgus-Kutrowska B, Bzowska A (2017) 1, $\mathrm{N}^{6}$-ethenoadenine and other fluorescent nucleobase analogues as substrates for purine-nucleoside phosphorylases: spectroscopic and kinetic studies. Curr. Pharm. Des. 23: 69726990. https://doi.org/10.2174/1381612823666171011103551

Yehia H, Kamel S, Paulick K, Neubauer P, Wagner A (2017) Substrate spectra of nucleoside phosphorylases and their potential in the production of pharmaceutically active compounds. Curr. Pharm. Design 23: 6913-6935. https://doi.org/10.2174/1381612823666171024155 811 